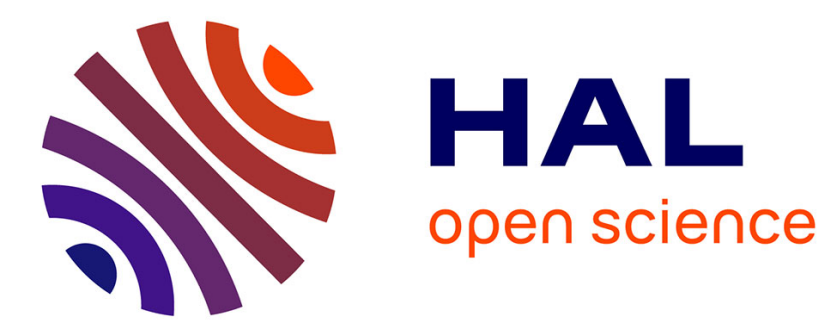

\title{
Structure and diffusion in aluminium and gallium trihalide melts from simulations based on intramolecular force laws
}

Giorgio Pastore, Romina Ruberto, Zehra Akdeniz, Mario Tosi

\section{- To cite this version:}

Giorgio Pastore, Romina Ruberto, Zehra Akdeniz, Mario Tosi. Structure and diffusion in aluminium and gallium trihalide melts from simulations based on intramolecular force laws. Molecular Physics, 2008, 105 (17-18), pp.2383-2392. 10.1080/00268970701651706 . hal-00513143

\author{
HAL Id: hal-00513143 \\ https://hal.science/hal-00513143
}

Submitted on 1 Sep 2010

HAL is a multi-disciplinary open access archive for the deposit and dissemination of scientific research documents, whether they are published or not. The documents may come from teaching and research institutions in France or abroad, or from public or private research centers.
L'archive ouverte pluridisciplinaire HAL, est destinée au dépôt et à la diffusion de documents scientifiques de niveau recherche, publiés ou non, émanant des établissements d'enseignement et de recherche français ou étrangers, des laboratoires publics ou privés. 


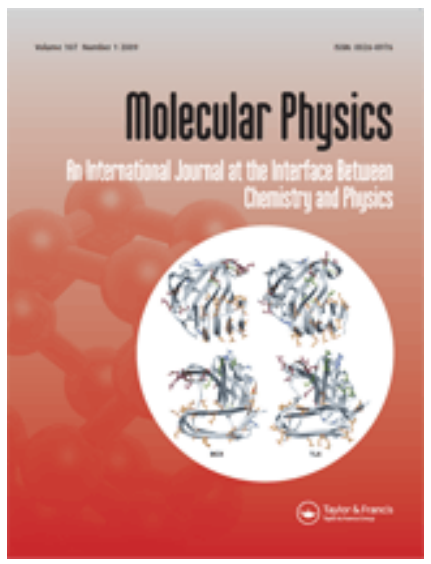

\section{Structure and diffusion in aluminium and gallium trihalide melts from simulations based on intramolecular force laws}

\begin{tabular}{|r|l|}
\hline Journal: & Molecular Physics \\
\hline Manuscript ID: & TMPH-2007-0169.R1 \\
\hline Manuscript Type: & Full Paper \\
\hline Date Submitted by the \\
Author: & 23-Aug-2007 \\
\hline Complete List of Authors: & $\begin{array}{l}\text { Pastore, Giorgio; Università di Trieste, Dept. of Theoretical Physics; } \\
\text { CNR-INFM, DEMOCRITOS National Simulation Centre } \\
\text { Ruberto, Romina; CNISM, Unità di Messina; CNR-INFM, } \\
\text { DEMOCRITOS National Simulation Centre } \\
\text { Akdeniz, Zehra; Istanbul University, Dept. of Physics } \\
\text { Tosi, Mario; Scuola Normale Superiore }\end{array}$ \\
\hline Keywords: & $\begin{array}{l}\text { Molten salts, Molecular dynamics, Molecular liquids, Polarizable } \\
\text { potentials }\end{array}$ \\
\hline \multicolumn{2}{|c}{} \\
\hline
\end{tabular}

\section{$\diamond$ ScholaroNE" \\ Manuscript Central}




\title{
Structure and diffusion in aluminium and gallium trihalide melts from simulations based on intramolecular force laws
}

\author{
By R. RUBERTO ${ }^{1,2}$, G. PASTORE ${ }^{1,3}$, Z. AKDENIZ $^{4}$ and M. P. TOSI ${ }^{5}$ \\ ${ }^{1}$ CNR-INFM DEMOCRITOS National Simulation Center, I-34100 Trieste, Italy \\ ${ }^{2}$ CNISM and Dipartimento di Fisica dell'Università, I-98166 Messina, Italy \\ ${ }^{3}$ Dipartimento di Fisica Teorica dell’Università, I-34100 Trieste, Italy \\ ${ }^{4}$ Physics Department, Istanbul University, 34118 Vezneciler-Istanbul, Turkey \\ ${ }^{5}$ Scuola Normale Superiore, I-56126 Pisa, Italy
}

Diffraction studies of the liquid structure of $\mathrm{AlCl}_{3}, \mathrm{AlBr}_{3}, \mathrm{GaBr}_{3}$ and $\mathrm{GaI}_{3}$ close to their respective freezing points have revealed fourfold coordination of the trivalent metal ions, consistent with dimeric $\mathrm{M}_{2} \mathrm{X}_{6}$ molecules being the dominant species. We evaluate the species-resolved pair distribution functions and liquid structure factors in all these melts by carrying out classical molecular-dynamics simulations, based on polarizablehalogen force laws that were determined on isolated molecular monomers and dimers in the gaseous phase. We also report results for mean-square displacements and diffusion coefficients of the two species in each melt. The model reproduces the main features of the total neutron-diffraction structure factors, showing peaks due to intermediate-range order and to charge and density short-range order, and accounts for the experimental data at a good quantitative level. Direct simulation of the pair distribution functions yields agreement with the diffraction data on metal-halogen and halogen-halogen bond lengths in the melt and on the stability of the first-neighbor shell of the metal ions. We examine the temperature dependence of liquid structure in our models for $\mathrm{GaBr}_{3}$ and $\mathrm{AlCl}_{3}$ and emphasize the structural role of van der Waals interactions between the halogens.

PACS numbers: 61.20.Qg - Structure of associated liquids: electrolytes, molten salts 61.25.Em - Molecular liquids 


\section{Introduction}

Trivalent-metal halide salts present an interesting variety of melting mechanisms, which results from a competition between (i) network formation in a cation-dominated Coulomb-ordered liquid and (ii) stabilization of molecular-type liquid structures with strong intermolecular correlations [1]. The crystal structures of the salts of present interest are commonly characterized as slightly distorted close-packed (cubic or hexagonal) arrangements of the halogens, within which the metal ions can occupy alternate layers of either octahedral or tetrahedral sites [2]. Tetrahedrally coordinated crystal structures as realized in $\mathrm{AlBr}_{3}$ and $\mathrm{GaBr}_{3}$ can alternatively be described as formed from ordered arrangements of $\mathrm{M}_{2} \mathrm{X}_{6}$ molecular dimers [2], and these basic structural units have been observed in neutron diffraction experiments to be stable across melting in these two compounds [3]. Sixfold coordination also is usually stable across melting, leading to loose-network structures as in the series of lanthanide-metal halides, but a remarkable exception is presented by $\mathrm{AlCl}_{3}$, which from an $\mathrm{X}$-ray diffraction study has been reported to melt from an ionic layer structure into a molecular liquid of $\mathrm{Al}_{2} \mathrm{Cl}_{6}$ dimers [4]. Measurements of the total neutron diffraction pattern of molten $\mathrm{AlCl}_{3}$ [5] have confirmed the fourfold coordination of the metal ions. Finally, $\mathrm{GaI}_{3}$ crystals have been variously reported as having a layer structure with octahedral coordination or a dimerized molecular structure, but again the $\mathrm{Ga}_{2} \mathrm{I}_{6}$ dimers are observed to be the majority species in the melt [3]. These various melting behaviors are signaled by the values of the thermodynamic melting parameters (melting temperature and melting entropy and volume) and of the ionic conductivity of the melt [6].

Electron diffraction on the gaseous phase has been used to determine the structure of isolated molecules of trihalide materials [7]. Both trigonal-planar $\mathrm{MX}_{3}$ monomers and $\mathrm{M}_{2} \mathrm{X}_{6}$ dimers are usually met, the latter consisting of two edge-sharing tetrahedra, 
but $\mathrm{GaI}_{3}$ has only been found as a monomer [8]. Dimerization in molten $\mathrm{GaI}_{3}$ could be incomplete, of order 70-80\% [3], as is also indicated by Raman-scattering data [9].

We present in this work the results of classical molecular-dynamics runs focusing on how well an ionic-interaction law determined from properties of isolated molecular clusters in the gas fares in regard to the observed pair structure from the neutron diffraction experiments on the liquid phase of $\mathrm{AlCl}_{3}, \mathrm{AlBr}_{3}, \mathrm{GaBr}_{3}$ and $\mathrm{GaI}_{3}$ near freezing $[3,5]$. The interionic force law is taken from the work of Akdeniz et al. [6], who carried out a systematic study of the binding and the vibrational frequencies of monomeric and dimeric units of $\mathrm{Al}, \mathrm{Fe}$ and $\mathrm{Ga}$ chlorides, bromides and iodides. They also considered, in addition to neutral molecular states, ionized states formed by stripping or adding a halogen ion to a molecular unit. Thus the present approach not only allows for ionization processes leading to a finite ionic conductivity in these melts in standard thermodynamic conditions, but will permit extensions to studies of such melts in far-removed thermodynamic states in which large increases in temperature and pressure may induce breakage of dimers and ultimately lead to full ionization. This is in contrast with the common approach to molecular liquids in terms of intermolecular force laws acting between stable molecular units.

Recently, ab-initio molecular dynamics techniques have been applied to numerical simulation of molten $\mathrm{AlCl}_{3}[10,11]$. It is natural to ask whether there is any role left for model interaction studies like the present one. We believe that the answer is positive for at least two reasons. The first is that even a computationally heavy force field like the present polarizable-ion model is two or three orders of magnitude faster than ab-initio calculations for the same system. Well tuned model calculations, although less reliable than ab-initio studies, allow fast and extensive exploration of parameter space. The 
second reason is that model force fields try to identify a few relevant physical mechanisms as the main cause of the observed behavior. Thus, studying how well approximate models fare is complementary to ab-initio studies in order to understand how ionic interactions control physical properties. Finally, we note that identifying accurate force fields is a prerequisite for exploiting recent numerical simulation techniques combining in a symbiotic way model interactions and ab-initio methods $[12$, 13].

The plan of the paper is briefly as follows. The essential aspects of the model are recalled in section 2, which also gives some details on the simulation method using microcanonical molecular dynamics. We emphasize the special difficulties that we meet in following the evolution of a sample of a molecular liquid starting from its component ions, and the sensitivity of this evolution to thermodynamic conditions. Section 3 reports our main results for the pair structure of the melts and compares them with the available diffraction data, while section 4 describes the single-particle ionic motions in these molecular liquids by giving data for mean-square displacements and self-diffusion coefficients. We also illustrate in these two sections the role of the thermodynamic conditions of the sample by reporting pair structure and self-diffusion in our model for $\mathrm{GaBr}_{3}$ at various temperatures and fixed density, as well as by following the evolution with temperature of the liquid structure and the average cluster distribution in $\mathrm{AlCl}_{3}$. The role of van der Waals interactions between the halogens is also discussed in the latter connection. The paper ends with a summary and some discussion of future perspectives in section 5 .

\section{Interionic force law and simulation procedure}

The ionic interactions in our model are constructed from Born-Mayer-type pair 
potentials supplemented by shell-model dipoles located on the halogens. The inclusion of ion-core polarization created on the halogens both from electrical induction and from overlap deformability is indeed crucial to quantitatively emulate chemical bonding in binary ionic systems. We refer specifically in this connection to the recent work of Karaman et al. on the $\mathrm{NaCl}$ monomer [14], showing excellent quantitative agreement of such a model with measured molecular properties at equilibrium and with the potential energy curve of the molecule as determined by configuration-interaction quantumchemical calculations over a wide range of internuclear distance around equilibrium.

We write the potential energy $U\left(\left\{\mathbf{r}_{i j}\right\},\left\{\mathbf{p}_{i}\right\}\right)$ of the liquid simulation sample, which depends on all interionic bond vectors $\mathbf{r}_{i j}$ and on all electronic dipoles $\mathbf{p}_{i}$ carried by the halogens, as

$$
\begin{aligned}
U\left(\left\{\mathbf{r}_{i j}\right\},\left\{\mathbf{p}_{i}\right\}\right) & =\sum_{i<j}\left[\frac{z_{i} z_{j} e^{2}}{r_{i j}}+\Phi_{i j}\left(r_{i j}\right)-\frac{C_{i} C_{j}}{r_{i j}^{6}}\left(1-e^{-\left(r_{i j} / \xi\right)^{6}}\right)\right] \\
& +U_{p o l}^{c l}\left(\left\{\mathbf{r}_{i j}\right\},\left\{\mathbf{p}_{i}\right\}\right)+U_{\text {shell }}\left(\left\{\mathbf{r}_{i j}\right\},\left\{\mathbf{p}_{i}\right\}\right)
\end{aligned}
$$

The sum in equation (1) includes (i) the Coulomb energy of the ionic point charges having nominal valences $z_{i}$, (ii) the overlap repulsive energy as described by the Busing form [14]

$$
\Phi_{i j}(r)=f\left(\rho_{i}+\rho_{j}\right) \exp \left[\left(R_{i}+R_{j}-r\right) /\left(\rho_{i}+\rho_{j}\right)\right]
$$

in terms of transferable radii $R_{i}$ and stiffness parameters $\rho_{i}$, and (iii) the van der Waals energy of the halogens. In regard to the latter, we have eliminated the risk of sample collapse from the imbalance between inverse-sixth-power attractions and exponential repulsions in close halogen-halogen collisions by the simple expedient of inserting a continuous saturation factor involving a parameter $\xi$. The electronic polarization of the 
halogens is allowed in equation (1) through both the classical polarization energy $U_{p o l}^{c l}$ and the shell deformation energy $U_{\text {shell }}$, the latter being patterned after the shell model used in the lattice dynamics of ionic and semiconducting crystals [16]. Explicit expressions for these energy terms can be found in Ref. [17]. It was also shown there that the results for the bond lengths in isolated monomers and dimers are essentially unchanged when the nominal ionic valences are replaced by effective valences, provided that use is made of the equilibrium condition in redetermining the overlap repulsive parameters. Saturation of van der Waals attractions at short range as included in equation 1 is, of course, consistent with the saturation of the effective ionic polarizability that is embodied in the shell model.

Minimization of equation (1) with respect to the halogen dipoles in any given configuration of the ionic positions yields the dipole $\mathbf{p}_{h}$ on the $h$-th halogen as the sum of a classical point-ion term proportional to the local electric field and of a counteracting dipole due to the change in the overlap of the valence electron shell of the halogen with the neighboring metal ions,

$$
\mathbf{p}_{h}=\alpha_{h} \mathbf{E}_{h}\left(\left\{\mathbf{r}_{i j}\right\},\left\{\mathbf{p}_{i}\right\}\right)+\alpha_{s} \sum_{i_{j}} \hat{\mathbf{r}}_{i h}\left|\frac{d \Phi_{i h}\left(r_{i h}\right)}{d r_{i h}}\right| .
$$

Here, $\mathbf{E}_{h}$ is the self-consistent electric field on the halogen, $\alpha_{h}$ and $\alpha_{s}$ are its electrical and short-range polarizabilities, and the sum is restricted to run over its first-neighbor metal ions. We have taken the values of the force-law parameters from the analysis of isolated molecular structures given in Ref. [6]. The precise value of the parameter $\xi(\approx$ $0.2-0.3 \mathrm{~nm}$ ) is largely arbitrary, within a range lying between the metal-halogen and halogen-halogen bond lengths. On inclusion of saturation, the calculated bond lengths in the isolated dimers are then affected by $0.004 \mathrm{~nm}$ in the worst case.

With the potential energy of the ionic assembly written as a function of the bond 
vectors and of the halogen dipoles, we perform standard microcanonical moleculardynamics calculations using Beeman's algorithm [18]. Our code implements a full calculation of Ewald sums for the long-range (charge-charge, charge-dipole, and dipoledipole) interactions, allowing full accuracy control in the calculation of energy and forces. The linear system of equations (3) for the halogen dipoles is numerically solved at each time step: this strategy is quite inefficient, but we have ascertained that a sample of 256 ions under periodic boundary conditions already suffices to yield reliable results for liquid structure in the thermodynamic states of present interest, and still allows us to carry out very long simulations on a personal computer. We have shown elsewhere for $\mathrm{AlCl}_{3}[19]$ that this sample size yields results that in practice are indistinguishable from those obtained on smaller samples of 108 ions.

Long runs and ample statistics are generally required in order to follow the evolution of each sample from the assembly of its component ions towards a molecular liquid structure and beyond. The simulation protocol handling these problems is essentially the same for all model liquids. We initialize the sample with the ions on a face-centered cubic lattice at the experimental density inside a cubic box with periodic boundary conditions and optimize the ionic positions by a sequence of $10^{4}$ steepestdescent steps. After melting and equilibrating the sample at $3000 \mathrm{~K}$, we cool it to the temperature of the neutron diffraction experiments and equilibrate it for at least 48 ps. The statistics is then collected by sampling at least 6000 configurations in a microcanonical run over an interval of at least 48 ps. A time step of 2 fs within Beeman's algorithm ensures excellent conservation of the energy. Longer runs and greater statistics are taken as needed in some cases, and the role of the thermodynamic state of the sample will be illustrated by showing results for the temperature dependence 
of liquid-state properties for $\mathrm{GaBr}_{3}$ and $\mathrm{AlCl}_{3}$.

Our explicit aim has been to understand how force-field parameters, tuned on cluster properties, fare in describing bulk liquids, and we can anticipate deviations from the true equation of state. However, it is reassuring to find that the estimated pressure of our simulation samples, at the experimental densities are in the range of a GPa, admittedly high but close to the values reported by Hutchinson et al. [20] for $\mathrm{AlCl}_{3}$. Simulation runs at the experimentally known atmospheric-pressure density are standard practice to ensure that the length scale is correctly fixed for comparisons between simulation and measurement of liquid structure.

The main output of the calculation are the partial pair distribution functions $g_{i j}(r)$ and the partial structure factors $S_{i j}(k)$, with each suffix denoting an ionic species. The former are obtained from counting interionic distances in $\mathbf{r}$-space and the latter are determined by a direct method using the correlations between partial ionic density fluctuations of wave vectors $\mathbf{k}$ and -k. Comparisons with the structure factors obtained from the Fourier transform of $g_{i j}(r)$ have been shown for $\mathrm{AlCl}_{3}$ elsewhere [19]. The selfdiffusion coefficients of the two ionic species are obtained from their mean square displacements through the Einstein relation.

\section{Results for liquid structure}

\subsection{Total neutron structure factor and partial structure factors}

Figure 1 reports our results for the neutron-weighted structure factor $S(k)$ of trihalide melts near freezing at the experimental density $\rho$. Here and in the following we adopt the definitions of Ref. [3], and in particular $S(k)$ is defined as

$$
S(k)=\left[b_{M}^{2} S_{M M}^{(F Z)}(k)+6 b_{M} b_{X} S_{M X}^{(F Z)}(k)+9 b_{X}^{2} S_{X X}^{(F Z)}(k)\right] /<\bar{b}>^{2} .
$$


Here $\mathrm{M}$ represents $\mathrm{Al}$ or $\mathrm{Ga}$ and $\mathrm{X}$ represents $\mathrm{Cl}$ or $\mathrm{Br}$ or $\mathrm{I}$ as appropriate, $b_{i}$ are the coherent neutron scattering lengths, $<\bar{b}>=b_{M}+3 b_{X}$, and $S_{i j}^{(F Z)}(k)$ are the Faber-Ziman partial structure factors. These we evaluate as $S_{i j}^{(F Z)}(k)=N^{-1}<\delta \rho_{i}(\mathbf{k}) \delta \rho_{j}(-\mathbf{k})>$, with $N$ the total number of ions and $\delta \rho_{i}(\mathbf{k})$ the density fluctuation of given wave vector $\mathbf{k}$ for each species. In figure 1 we also report the neutron-diffraction data for the two melts [3, 5]. It should be remarked that the values of the Faber-Ziman coefficients in equation (4), as reported in $[3,5]$, are such that the neutron-weighted diffraction pattern is quite insensitive to the M-M correlations, and especially so in the Al salts. Simulation results for the Ashcroft-Langreth partial structure factors $S_{i j}(k)$ of the models for the $\mathrm{AlCl}_{3}$ and $\mathrm{AlBr}_{3}$ melts are shown in figure 2. The partial structure factors of the other melts show entirely similar features.

The three main peaks shown by $S(k)$ in figure 1 as $k$ increases correspond, as is clear from comparison with the main features in the partial structure factors in figure 2 , to intermediate-range order and to charge and density short-range order. In fact, there also is a contribution from density-density correlations to the second peak in $S(k)$. The comparison of our results with the neutron diffraction data shows some discrepancies of detail mainly in the height of some peaks, but the overall agreement is good. This degree of agreement between models and experiments crucially depends on the inclusion of van der Waals interactions, and we take it to be a confirmation of a molecular-like state of short-range structural order. In fact, van der Waals interactions have an important role in determining the shape of the average distribution of ion groups, which in addition to a majority of dimers contains an appreciable fraction of trimers as well as (especially in $\mathrm{AlBr}_{3}$ and $\mathrm{GaI}_{3}$ ) an appreciable fraction of recognizably single monomers. We also remark that, as already discussed in Ref. [19], there is good agreement between our 
results for $\mathrm{AlCl}_{3}$ and those of the earlier simulations by Hutchinson et al. [20] for both the neutron-weighted and the partial structure factors: their simulation samples were considerably larger and their model was to some extent adjusted to data on the melt.

A further point to be emphasized from the results shown in figures 1 and 2 is the great structural similarity of the $\mathrm{AlCl}_{3}$ melt with the $\mathrm{AlBr}_{3}$ and $\mathrm{GaBr}_{3}$ melts. It is rather well established for the two latter systems that they possess a molecular-type structure based on $\mathrm{M}_{2} \mathrm{X}_{6}$ dimers both in the crystalline phase and in the melt. Our results thus provide further evidence that the $\mathrm{AlCl}_{3}$ melt near freezing also possesses such dimerbased structure.

\subsection{Radial distribution functions}

The full lines in figure 3 show our results for the neutron-weighted pair distribution function (pdf) $G(r)$ of trihalide-melt models at the same temperature and density as in figure 1 . The dotted lines in figure 3 report for comparison the corresponding neutron-weighted pdf obtained by Fourier inversion of the measured $S(k)$ in the neutron scattering experiments [3,5]. The simulation results for $G(r)$ have instead been constructed from partial pdf's $g_{i j}(r)$ according to the definition $G(r)=$ $1+\sum_{i, j} c_{i} c_{j} b_{i} b_{j}\left[g_{i j}(r)-1\right] /<\bar{b}>^{2}$, where $c_{i}$ are the concentrations of the two species.

The partial pdf's were obtained from the simulation runs by statistical averaging of direct counts of ion-pair distances in the sample. Those shown in figure 4 for $\mathrm{AlBr}_{3}$ are representative of all model trihalide liquids. In figure 5 we show our results for the running coordination numbers $N_{i j}(r)$ of metal ions and halogen ions, defined as $N_{i j}(r)=4 \pi n_{j} \int_{0}^{r} g_{i j}(s) s^{2} d s$ where $n_{j}$ are the partial average densities. In all cases we find that a dimer-like configuration yielding plateaus at $N_{M X}(r)=4$ and $N_{M M}(r)=1$ is the 
majority species, but at times we can observe during the evolution of the sample the formation of higher n-mer states.

The local liquid-state order revealed by the neutron-weighted pdf in figure 3 shows three distinctive features in all melts: (i) a first-neighbor peak, which is associated with the $\mathrm{M}-\mathrm{X}^{\mathrm{T}}$ bond length in a dimer (with $\mathrm{X}^{\mathrm{T}}=$ terminal halogen) and lies at a position in very close agreement between simulation models and neutron diffraction experiments; (ii) a second main peak, which is mainly associated with the $\mathrm{X}^{\mathrm{T}}-\mathrm{X}^{\mathrm{T}}$ bond length in the dimer and with inter-dimer $\mathrm{X}^{\mathrm{T}}-\mathrm{X}^{\mathrm{T}}$ correlations, and closely agrees in location with the neutron diffraction data; and (iii) a deep trough between these two peaks, where the low probability of finding ions indicates that the exchange of ions between the first-neighbor shell and the rest of the liquid is very low, as is the case for a stable molecular-type first-neighbor coordination. A fourth feature in the $G(r)$ of the simulation models is the small peak or shoulder on the right side of the first main peak, which is associated with the $\mathrm{M}-\mathrm{X}^{\mathrm{B}}$ bond length in the dimer (with $\mathrm{X}^{\mathrm{B}}=$ bonding halogen). In this region the neutron-diffraction data show only skewness in the firstneighbor peak. The data of Saboungi et al. for the heavy-halogen melts [3] show, however, a small bump in $G(r)$ further out, where no feature is visible in the simulation models.

The above statements are quantified by reporting in Table 1 the main nearneighbor bond lengths in liquid $\mathrm{MX}_{3}$ from the simulation models and from the neutron and X-ray diffraction experiments on the melts, in comparison with those measured for the isolated molecular dimer by electron diffraction on the gas [7, 21]. The SML bond lengths have been read from the partial pdf's obtained in the simulations, while the NDL bond lengths in the heavier halides have been reported by Saboungi et al. [3] as the results of a fit of their data by mans of the Reference Interaction Site Model (RISM) 
with adjustable parameters representing hard-sphere radii.

It is also evident from figure 3 that the sharpness of the first pair-correlation peaks is overemphasized in the simulation models. It should be stressed again at this point that the model evaluates the partial radial distribution functions by direct counting of bond lengths, whereas the experiments obtain $G(r)$ by Fourier inversion of the neutronweighted total structure factor. Quantitative faults may arise in the simulation model from various defects in the adjustment of its parameters to molecular data [6], such as the neglect of anharmonicity in molecular vibrations and of finite-temperature effects, or the omission of a possible minor reduction of the ionic valences below their nominal values as first proposed in Ref. [17]. Comparison of our data with experiment in the region to the right of the second peak of $G(r)$ indicate that experimental data in such region contain valuable information about atomic correlations beyond the intra-dimer level. The quantitative value of our results in this region crucially depends on the inclusion of van der Waals interactions.

On the other hand, we should also remark that the resolution of structures in $G(r)$ from Fourier inversion of $S(k)$ is affected by truncation of the data in momentum space. To examine this question we have evaluated $G(r)$ by Fourier inversion of $S(k)$ as obtained in the simulation model from the correlations of density fluctuations, after windowing the momentum-space correlations with a factor $\exp \left[-q^{2} /\left(2 \sigma^{2}\right)\right]$. The dashed lines in figure 3 show the result for the case $\sigma=150 \mathrm{~nm}^{-1}$. The dependence of the result on the value of $\sigma$ has been reported elsewhere [22], as an illustration of the challenge posed to neutron diffraction experiments on molecular liquids by the quantitative determination of sharp local structures in $\mathbf{r}$-space. A detailed analysis of the distributions of angles subtended by ion triplets in molecular liquids has also been given in Ref. [22]. 


\subsection{Temperature dependence of liquid structure in the model for $\mathrm{GaBr}_{3}$}

As we shall see in section 4 below, there is some indication from our simulation data on ionic diffusion that especially in $\mathrm{GaBr}_{3}$ the model parameters that we have adopted for the melt at the experimental temperature and density may be more appropriate to describe a supercooled liquid state.

We have carried out simulations on this model of $\mathrm{GaBr}_{3}$ at higher temperatures and at the same density. Figure 6 shows a comparison between results for $S(k)$ and $G(r)$ at $413 \mathrm{~K}$ and $1000 \mathrm{~K}$. The raise of temperature leaves the main features of these functions substantially unchanged, with some improvements in comparison with the experimental data. The effect of temperature on the ionic motions in $\mathrm{GaBr}_{3}$ will be illustrated later below.

\subsection{Temperature dependence of liquid structure in the model for $\mathrm{AlCl}_{3}$}

The simulation results in figure 7 illustrate instead the effect of a temperature increase on the structure of our model for $\mathrm{AlCl}_{3}$, as an example of the consequences to be expected from a change of thermodynamic state on a liquid which, in contrast to the $\mathrm{GaBr}_{3}$ model, appears to be well equilibrated near experimental freezing. In these runs we have allowed for thermal expansion by adopting the expression for the temperaturedependent density of the liquid reported by Janz [23], extrapolating it from its reported range of validity $(462 \mathrm{~K}<T<569 \mathrm{~K})$ up to $673 \mathrm{~K}$ in order to follow the evolution of the average cluster distribution as a function of temperature. This choice implies that the density of the model is almost halved on raising the temperature from $473 \mathrm{~K}$ to $673 \mathrm{~K}$.

The simulation results for the neutron-weighted structure factor in the top panel in figure 7 show that the main consequences of this temperature raise are located in the low-momentum region: the sample appears to be approaching the critical region of the 
model. The experimental liquid-gas critical point is located at $620 \mathrm{~K}$ and $0.52 \mathrm{~g} / \mathrm{cm}^{3}$. Our data on the average cluster composition of the melt reveal a clear trend towards dissociation of higher clusters into dimers. With increasing temperature some dimers may dissociate into monomers, but the extent of such dissociation is very sensitive to details of the interaction parameters through the relative cluster energies. In particular, the inclusion of van der Waals interactions tends to stabilize the dimer states relative to monomers in the liquid phase at intermediate densities, as may be realized if one views each dimer as "coated" by a shell of polarizable material inducing an effective shortrange attraction between dimers. To our knowledge, the only relevant experimental evidence refers to the dilute gaseous phase of $\mathrm{AlCl}_{3}$ : (i) the Raman scattering spectra show a transition from a mixture of dimers and monomers at the sublimation point to a purely monomeric gas at $900 \mathrm{C}$ [24]; and (ii) electron diffraction yields $29 \%$ dimers at $400 \mathrm{C}[25]$.

\section{Single-particle motions and self-diffusion coefficients}

Figure 8 reports our results for the mean-square displacement (msd) of the two ionic species in trihalide melts as functions of time. The corresponding values of the self-diffusion coefficients are obtained from the asymptotic slope of the msd and are collected in Table 2. Of course, it would be wholly inappropriate to estimate from these data the ionic conductivity in such mainly molecular liquids by means of the NernstEinstein relation, since diffusion is mostly associated with translational and rotational motions of neutral molecular units whereas ionic conduction requires ionization processes and hopping motions of halogen ions [26].

It is evident that in the present model the self-diffusion of both species in $\mathrm{GaBr}_{3}$, in the thermodynamic conditions that we have adopted, is especially slow. This behavior 
could be a signal of a supercooled liquid state, We have accordingly evaluated the ionic motions in this model as functions of temperature, the results being reported in figure 9 and Table 2. Clearly, normal diffusive behavior for a molecular liquid is recovered through such a temperature raise.

\section{Summary and future perspectives}

In summary, we have presented results from molecular dynamics simulation of the structure and the diffusive motions in models for group-IIIA halide melts, in comparison with data on liquid structure from neutron and X-ray scattering experiments. The simulation model has used force laws that were determined from properties of molecular monomers and dimers of these compounds in the gaseous phase, as described in terms of the interactions between the individual component ions. Of crucial importance is the inclusion of the electron-shell polarization of the halogen ions, which allows the model to emulate the formation of quantum chemical bonds. Our results show that one can get quite a reasonable description for structure and transport in the liquid phase of even complex ionic materials near freezing by means of a model whose parameters are determined from data on the gaseous phase. Improvements may be sought with the help of liquid-state data, the best candidate in this respect being the width of the first-neighbor shell that is expected to be very sensitive to the introduction of deviations from the nominal ionic valences.

To the extent that we have illustrated in our calculations, and bearing in mind that plenty of data are available in the literature on properties of the gaseous phase, the model has some predictive value for liquid-state properties. This will be especially useful for the estimation of structural and transport properties of these liquids on the approach to states that are expected to be met under extreme conditions of temperature and pressure, 
when the molecule-based liquid structure is broken up into that of an ionic liquid. These ionized states could be preceded by the transition from a predominantly dimeric to a monomeric liquid. Model studies will allow rapid exploration of such fluid-state transitions, to be ultimately examined by computer-expensive quantum mechanical methods.

For the specific materials that we have studied, our results provide additional evidence that the main species in their liquid structure near standard freezing is formed by stable molecular-dimer units with strong intermolecular correlations, and give new details on structure and diffusion by presenting species-resolved structure functions and mean-square displacements. Our data point to almost completely dimerized molecular fluids in near-freezing thermodynamic conditions, although fluctuations towards n-mer states and formation of monomer-dimer bonds can be observed in the course of the simulation runs. Our study of transport in the liquid phase should be extended to other properties such as the ionic conductivity and the deviations from the Nernst-Einstein relation, that would directly reflect the correlated motions of the two ionic species.

We acknowledge the contribution given by Enrico Goat to software development. One of us (R.R.) thanks CNISM - Unità di Messina for a grant supporting her stay at the Dipartimento di Fisica Teorica dell'Università di Trieste in the final part of this work. Z.A. acknowledges support from TUBITAK and from the Research Foundation of Istanbul University under Project Number BYP-846/07122005. Finally, Z.A. and M.P.T. thank Professor V. E. Kratsov and the Condensed Matter and Statistical Physics Section of the Abdus Salam International Center for Theoretical Physics in Trieste for their hospitality. 


\section{References}

[1] Tosi, M. P., Price, D. L., and Saboungi, M.-L., 1998, Ann. Rev. Phys. Chem., 44, 173.

[2] WyCKOFF, R. W. G., 1964, Crystal Structures, Vol, 2 (New York: Interscience).

[3] Saboungi, M.-L., Howe, M. A., and Price, D. L., 1993, Molec. Phys., 79, 847.

[4] Harris, R. L., WoOd, R. E., and RitTER, H. L., 1951, J. Amer. Chem. Soc., 73, 3150.

[5] Badyal, Y. S., Allen, D. A., and Howe, R. A., 1994, J. Phys.: Condens. Matter, 6, 10193.

[6] Akdeniz, Z., Pastore, G., and Tosi, M. P., 1998, N. Cimento, 20D, 595.

[7] Chase, M. W., Davies, C. A., Downey, J. R., Frurip, D. J., McDonald, R. A., and Syverud, A. N., 1985, J. Phys. Chem. Ref. Data, 14, Suppl. No. 1.

[8] MorinO, Y., UKAJI, T., and ITO, T., 1966, Bull. chem. Soc. Japan, 39, 71.

[9] Alvarenga, A. D., SABoungi, M.-L., Curtiss, L. A., Grimsditch, M., and MCNeIL, L. E., 1994, Molec. Phys., 81, 409.

[10] Kirchner, B., Seitsonen, A.P., and Hutter, J., 2006, J. Phys. Chem. B, 110, 11475.

[11] East, A. L. L., and Hafner, J., 2007, J. Phys. Chem. B, 111, 5316.

[12] Csanyi, G., Albaret, T., Payne, M.C., and De Vita, A., 2004, Phys. Rev. Lett., 93, 175503.

[13] Tangney, P., and SCandolo, S., 2003, J. Chem. Phys., 119, 9673.

[14] Karaman, A., Akdeniz, Z., and Tosi, M. P., 2006, Phys. Chem. Liq., 44, 353.

[15] Busing, W. R., 1970, Trans. Am. Crystallogr. Ass., 6, 57.

[16] See e. g. CochrAN, A. R., 1971, Crit. Rev. Solid State Sci., $2,1$. 
[17] AKDENIZ, Z., and TOSI, M. P., 1999, Zs. Naturforsch., 54a, 180.

[18] Allen, M. P., and TILDESLEY, D. J., 1987, Computer Simulation of Liquids (New York: Oxford University Press).

[19] Akdeniz, Z., Ruberto, R., Pastore, G., and Tosi, M. P., 2007, Proc. XV Int. Symp. Molten Salts, ECS Transactions, in press. The van der Waals interactions were dropped in the simulation study reported in this work-

[20] Hutchinson, F., Walters, M. K., Rowley, A. J., and Madden, P. A., 1999, J. Chem. Phys., 110, 5821.

[21] SHEN, Q., 1974, Diss. Abstr. (Int.), 34, 3735.

[22] Goat, E., Ruberto, R., Pastore, G., Akdeniz, Z., and Tosi, M.P., Phys. Chem. Liq. in press. The models studied in this work did not include van der Waals interactions.

[23] JanZ, G. J., 1988, J. Phys. Chem. Ref. Data, 17, Suppl. No. 2.

[24] Blander, M., 1964, Molten Salts Chemistry, (New York: Academic) p. 664.

[25] Aarset, K., Shen, Q., Thomassen, H., Richardson, A.D., and HedBERG, K., 1999, J. Phys. Chem. A, 103, 1644.

[26] AKDENIZ, A., and TOSI, M.P., 1998, Z. Naturforsch., 53a, 960. 


\section{Figure captions}

Figure 1. The squares show the neutron-weighted structure factor $S(k)$ constructed from partial structure factors as illustrated in figure 2, for models of (from top to bottom) (i) molten $\mathrm{AlCl}_{3}$ at $T=473 \mathrm{~K}$ and $\rho=1.27 \mathrm{~g} / \mathrm{cc}$, (ii) molten $\mathrm{AlBr}_{3}$ at $T=413 \mathrm{~K}$ and $\rho=2.55 \mathrm{~g} / \mathrm{cc}$, (iii) molten $\mathrm{GaBr}_{3}$ at $T=413 \mathrm{~K}$ and $\rho=3.22 \mathrm{~g} / \mathrm{cc}$, and (iv) molten $\mathrm{GaI}_{3}$ at $T=520 \mathrm{~K}$ and $\rho=3.53 \mathrm{~g} / \mathrm{cc}$. The dashed lines report the measured $S(k)$ from the neutron diffraction experiments of Badyal et al. [5] and of Saboungi et al. [3].

Figure 2. Ashcroft-Langreth partial structure factors $S_{i j}(k)$ of the models for the $\mathrm{AlCl}_{3}$ melt (left) and for the $\mathrm{AlBr}_{3}$ melt (right) at the temperature and density indicated in figure 1. From top to bottom: halogen-halogen, Al-halogen, and Al-Al correlations.

Figure 3. Neutron-weighted pair distribution function $G(r)$ for models of trihalide melts $\left(\mathrm{AlCl}_{3}, \mathrm{AlBr}_{3}, \mathrm{GaBr}_{3}\right.$ and $\mathrm{GaI}_{3}$, from top to bottom) at the temperatures and densities adopted in figure 1 . The simulated pdf for assemblies of 256 ions is shown as solid lines, whereas the dashed lines illustrate the effect of a momentum cut-off in obtaining $G(r)$ from Fourier inversion of $S(k)$ (see text). The dotted lines report the pdf from Fourier inversion of the measured neutron-weighted diffraction patterns $[3,5]$.

Figure 4. Partial radial distribution functions of the present model for molten $\mathrm{AlBr}_{3}$.

Figure 5. Partial running coordination numbers for models of trihalide melts $\left(\mathrm{AlCl}_{3}\right.$, $\mathrm{AlBr}_{3}, \mathrm{GaBr}_{3}$ and $\mathrm{GaI}_{3}$, from top to bottom) at the temperatures and densities adopted in figure 1. Solid lines: cation-anion MX; dashed lines: anion-anion XX; dotted lines: cation-cation MM.

Figure 6. Illustrating the effect of temperature in simulations of thestructure of the $\mathrm{GaBr}_{3}$ model. Top panel: the results for $S(k)$ at $T=413 \mathrm{~K}$ given in figure 1 (empty 
squares) are compared with simulation results at $T=1000 \mathrm{~K}$ (filled circles) and with the neutron-diffraction data of Saboungi et al. [3] (dashed line). Bottom panel: the same for $G(r)$, the corresponding symbols being a full line, a dashed line, and a dotted line.

Figure 7. Illustrating the effect of temperature in simulations of the structure of the $\mathrm{AlCl}_{3}$ model. Top panel: the results for $S(k)$ at $473 \mathrm{~K}$ and $1.27 \mathrm{~g} / \mathrm{cc}$ (full line) are compared with those at $573 \mathrm{~K}$ and $1.0 \mathrm{~g} / \mathrm{cc}$ (dashed line) and those at $673 \mathrm{~K}$ and 0.73 $\mathrm{g} / \mathrm{cc}$ (dotted line). Bottom panel: the same for $G(r)$.

Figure 8. Mean square displacement (in $\mathrm{nm}^{2}$ ) of the two ionic species as a function of time $t$ (in ps) in the model for trihalide melts $\left(\mathrm{AlCl}_{3}, \mathrm{AlBr}_{3}, \mathrm{GaBr}_{3}\right.$ and $\mathrm{GaI}_{3}$, from top to bottom) at the temperature and density adopted in figure 1.

Figure 9. Mean square displacement (in $\mathrm{nm}^{2}$ ) of the two ionic species as a function of time $t$ (in ps) in the model for $\mathrm{GaBr}_{3}$ at two temperatures (473 K and $1000 \mathrm{~K}$ ) and density $\rho=3.22 \mathrm{~g} / \mathrm{cc}$. 
Table 1. Near-neighbor bond lengths in $\mathrm{MX}_{3}$ (in $\mathrm{nm}$ ) from our simulation model of the liquid (SML), from neutron diffraction (NDL) and X-ray diffraction (XDL) experiments on the liquid, and from electron diffraction experiments on the gas (EDG).

\begin{tabular}{|c|c|c|c|c|c|}
\hline & $M-X^{T}$ & $M-X^{B}$ & M-M & $\mathrm{X}^{\mathrm{T}}-\mathrm{X}^{\mathrm{T}}$ & $X^{B}-X^{B}$ \\
\hline \multicolumn{6}{|l|}{$\mathrm{AlCl}_{3}:$} \\
\hline SML & 0.206 & 0.225 & 0.324 & 0.357 & 0.317 \\
\hline NDL [5] & 0.21 & ----- & ----- & 0.35 & ----- \\
\hline XDL [4] & 0.206 & 0.234 & 0.328 & 0.365 & 0.328 \\
\hline EDG [7] & 0.2065 & 0.2252 & 0.321 & 0.364 & 0.316 \\
\hline \multicolumn{6}{|l|}{$\mathrm{AlBr}_{3}:$} \\
\hline SML & 0.222 & 0.240 & 0.333 & 0.383 & 0.350 \\
\hline NDL [3] & 0.221 & 0.237 & 0.313 & 0.387 & 0.356 \\
\hline EDG [17] & 0.222 & 0.241 & 0.334 & 0.390 & 0.348 \\
\hline \multicolumn{6}{|l|}{$\mathrm{GaBr}_{3}:$} \\
\hline SML & 0.227 & 0.241 & 0.348 & 0.387 & 0.34 \\
\hline NDL [3] & 0.226 & 0.242 & 0.320 & 0.395 & 0.363 \\
\hline EDG [17] & 0.225 & 0.245 & 0.343 & 0.404 & 0.349 \\
\hline \multicolumn{6}{|l|}{$\mathrm{GaI}_{3}:$} \\
\hline SML & 0.247 & 0.263 & 0.370 & 0.422 & $\approx 0.4$ \\
\hline NDL [3] & 0.245 & 0.263 & 0.328 & 0.447 & 0.411 \\
\hline
\end{tabular}


Table 2. Self-diffusion coefficients of trihalide models at the indicated temperatures (in units of $10^{-6} \mathrm{~cm}^{2} / \mathrm{s}$ ).

\begin{tabular}{lccccc}
\hline & $\begin{array}{c}\mathrm{AlCl}_{3} \\
(473 \mathrm{~K})\end{array}$ & $\begin{array}{c}\mathrm{AlBr}_{3} \\
(413 \mathrm{~K})\end{array}$ & $\begin{array}{c}\mathrm{GaBr}_{3} \\
(413 \mathrm{~K})\end{array}$ & $\begin{array}{c}\mathrm{GaBr}_{3} \\
(1000 \mathrm{~K})\end{array}$ & $\begin{array}{c}\mathrm{GaI}_{3} \\
(520 \mathrm{~K})\end{array}$ \\
\hline $\mathrm{D}_{\mathrm{M}}$ & 15.2 & 1.0 & 0.42 & 6.8 & 1.95 \\
$\mathrm{D}_{\mathrm{X}}$ & 20.1 & 1.8 & 0.60 & 9.4 & 2.67 \\
\hline
\end{tabular}




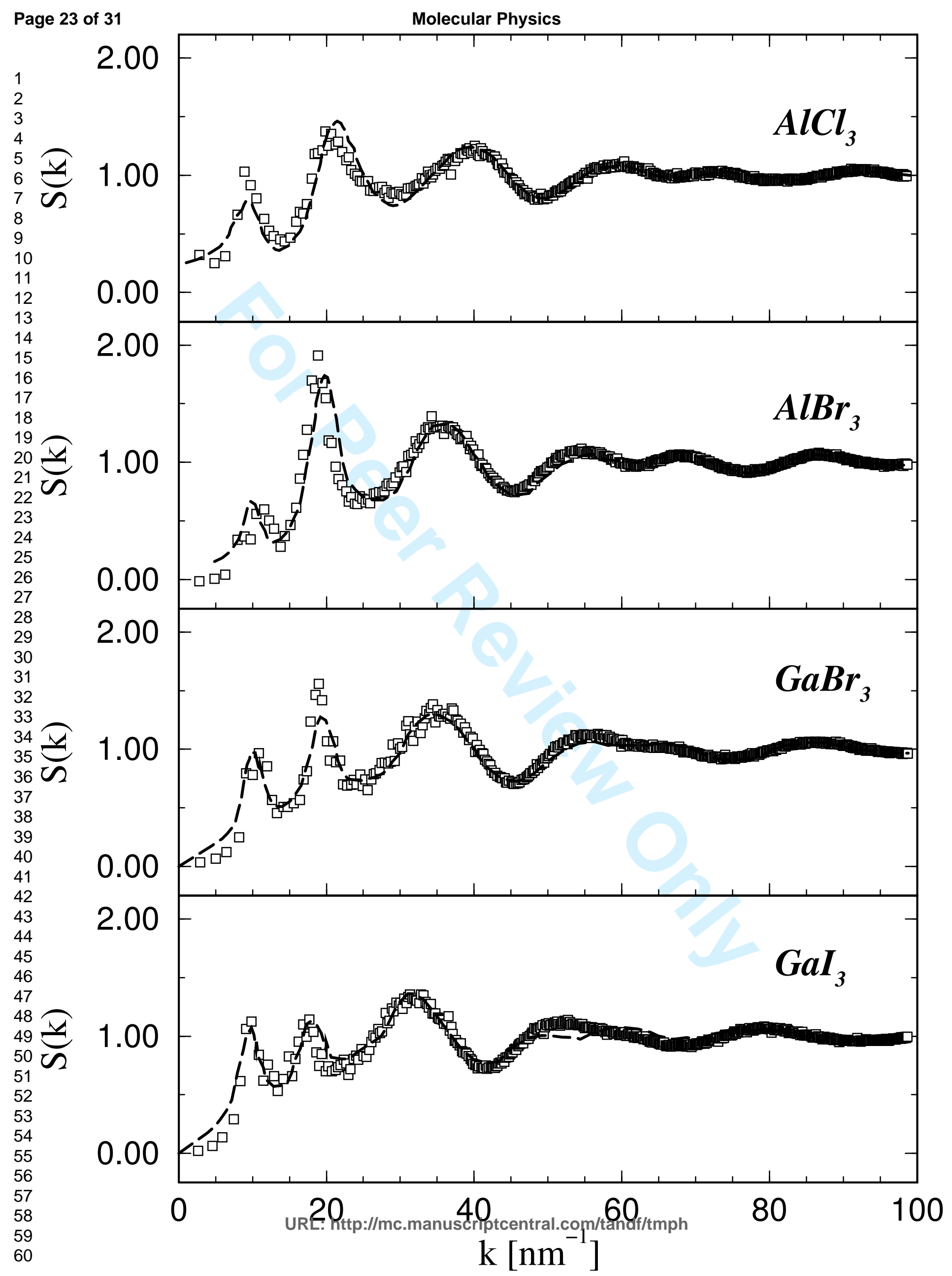




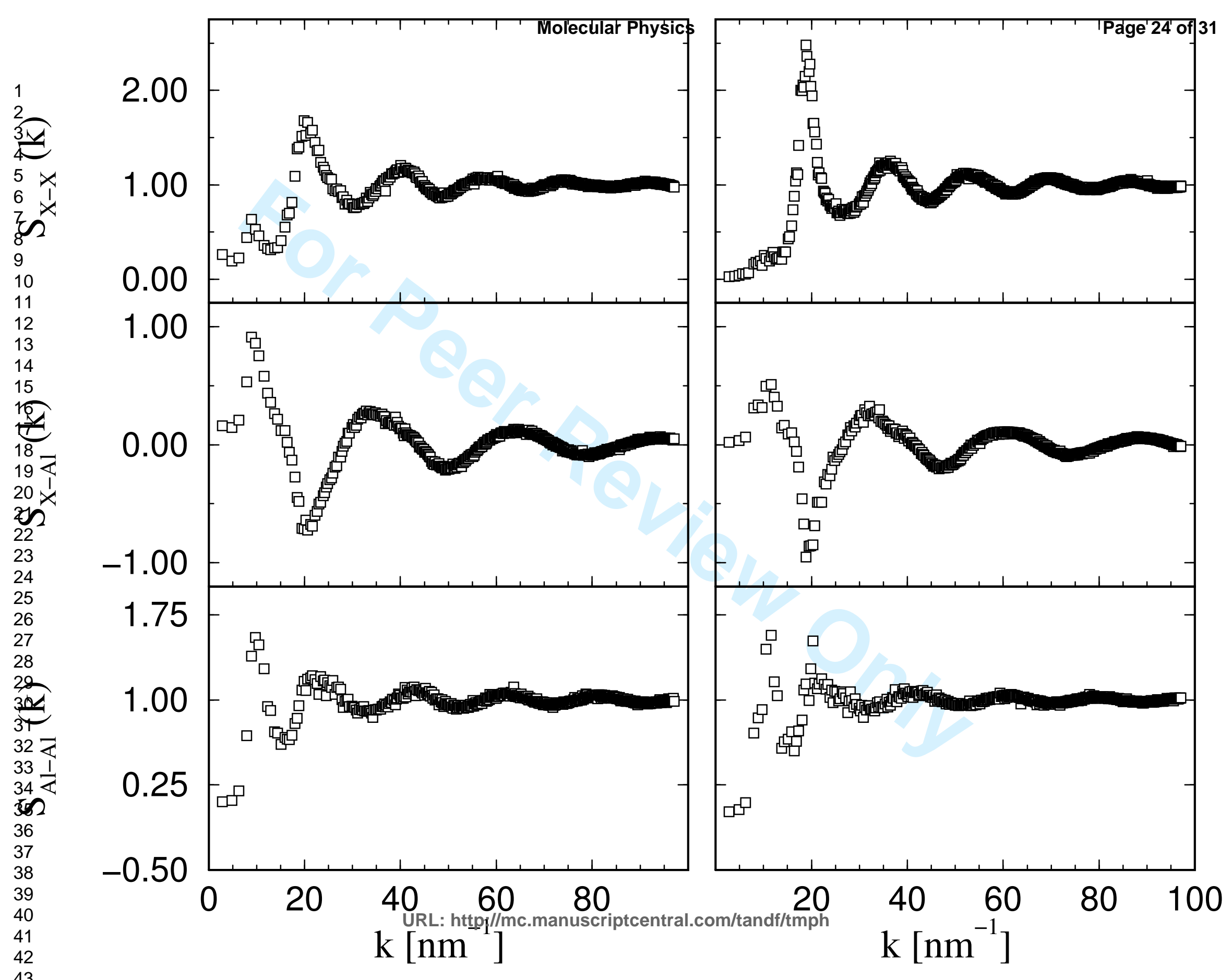




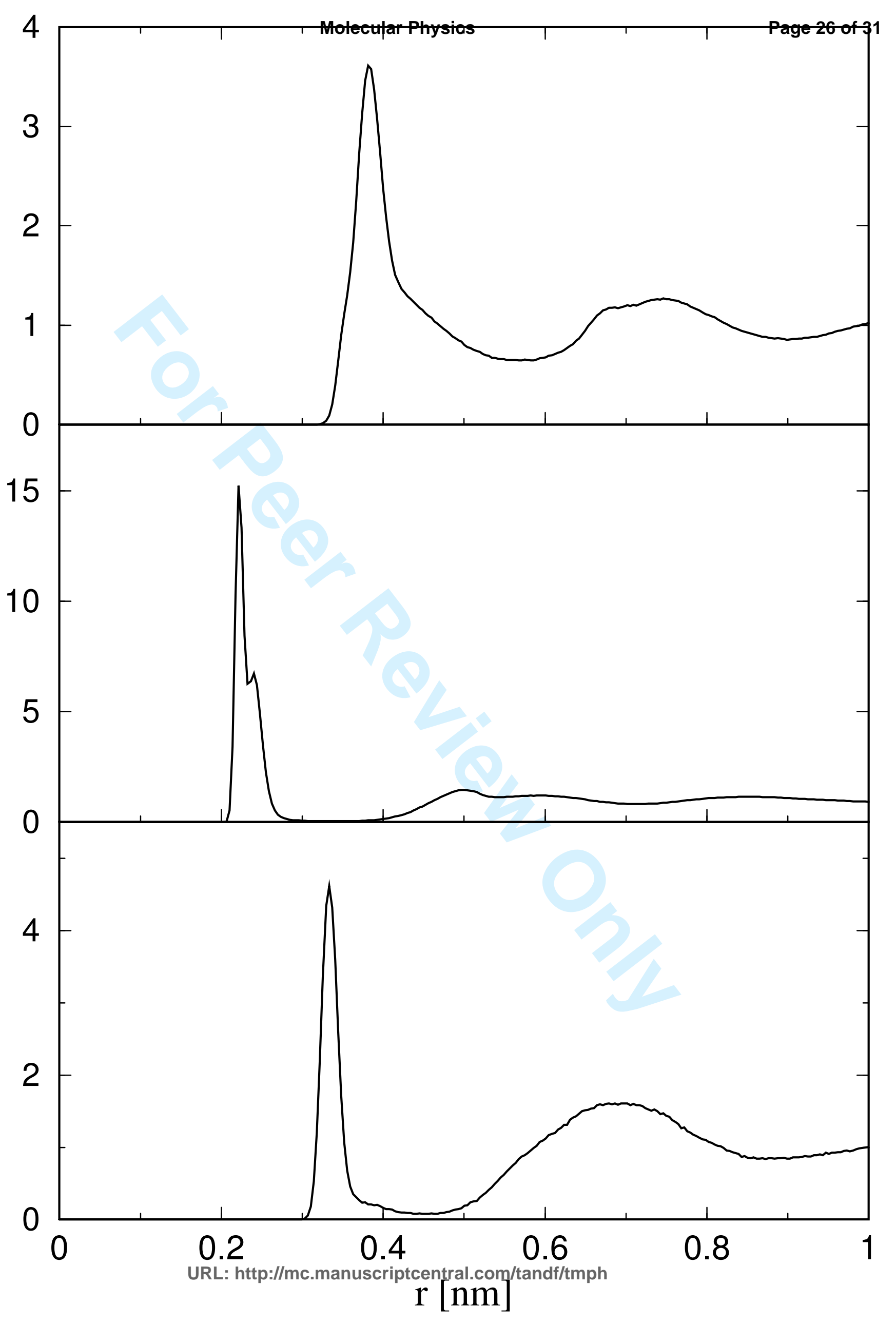




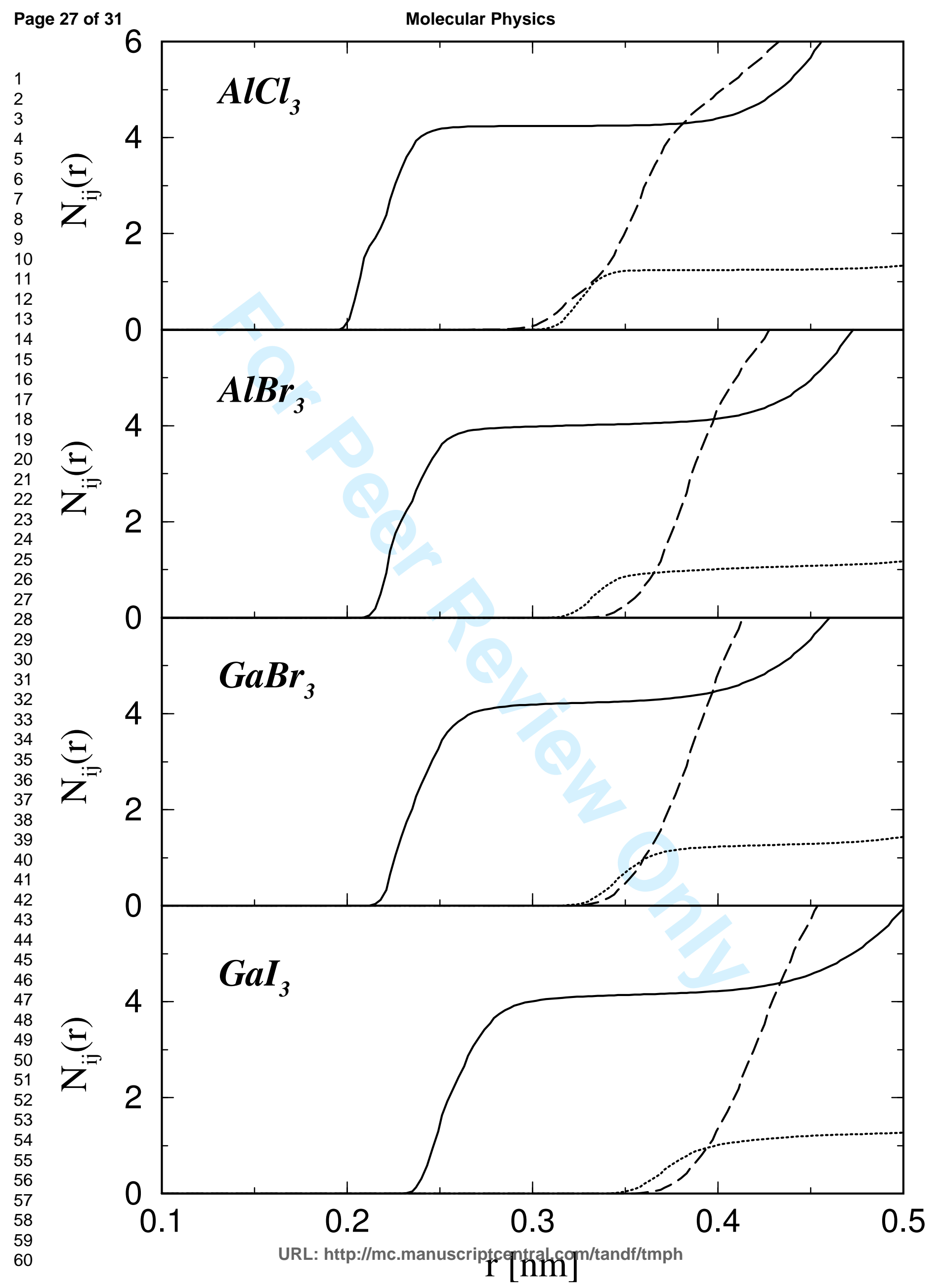




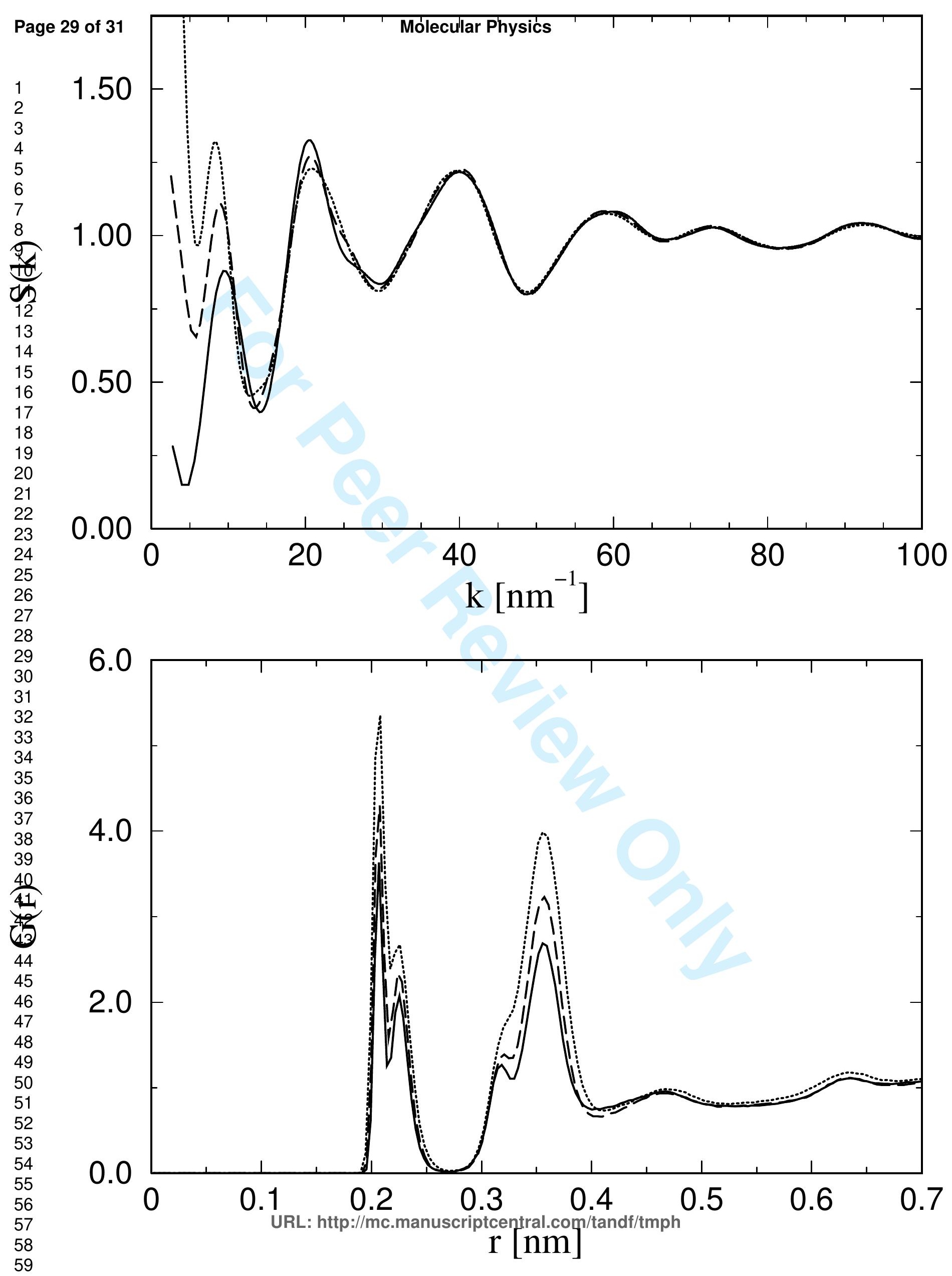




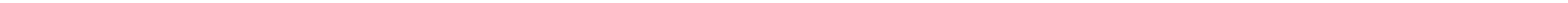




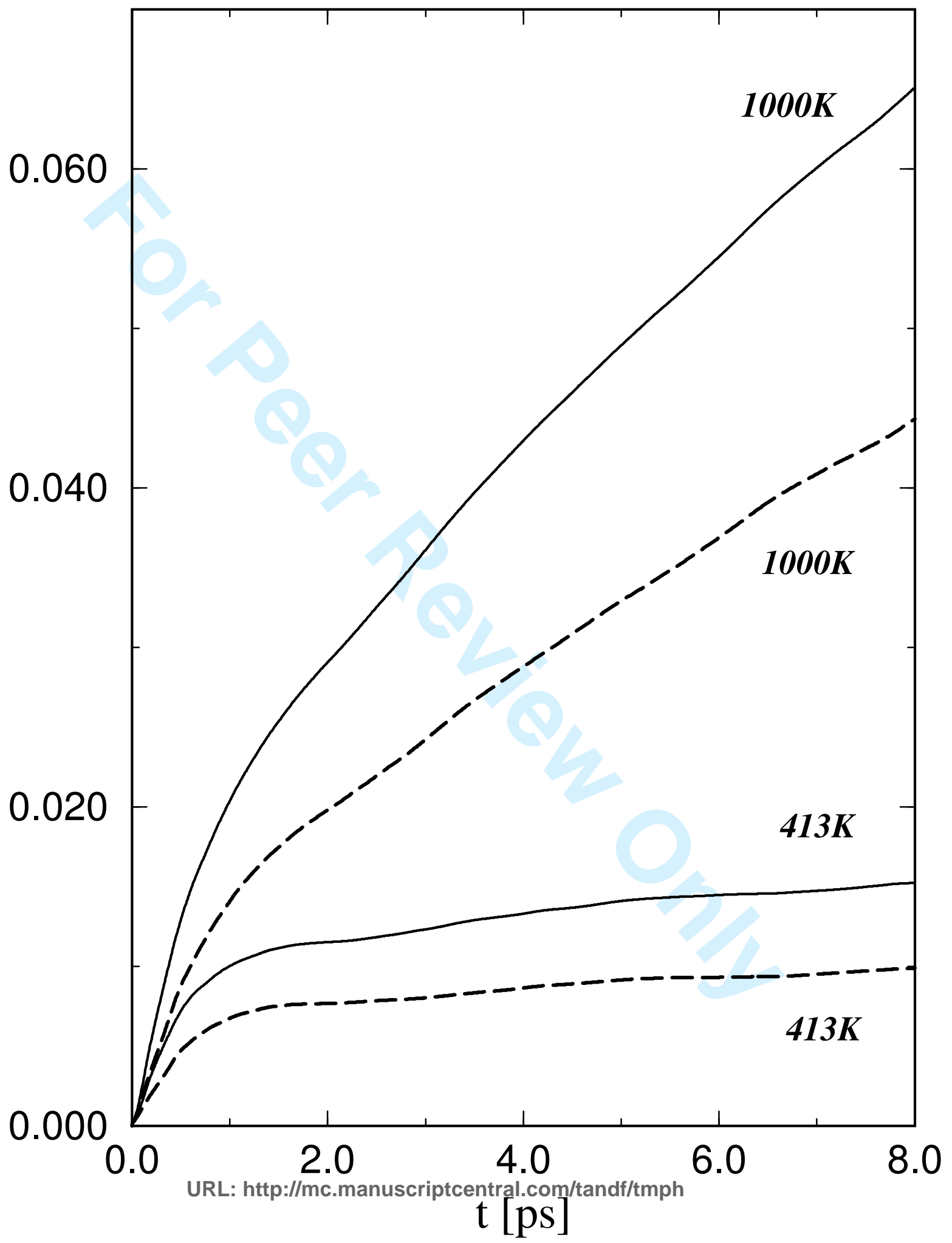

